\title{
Sphingolipids: Friends or Foes?
}

\author{
Hiroshi Hayashi
}

Department of Internal Medicine, Tokyo Ariake University of Medical and Health Sciences, Tokyo, Japan

\section{See article vol. 29: 000-000}

Sphingolipids play a physiologically major role in the human body. They not only serve as essential constituents of the membrane but also make microdomains called lipid rafts with cholesterol on the cell surface for transporting substances in and out of the cells. Sphingomyelin constitutes myelin sheath and red blood cell membrane. In contrast, if sphingomyelin is abnormally stored in the reticuloendothelial system and neurons of the body, lethal damage can occur after birth. This situation occurs in Niemann-Pick disease type A and B genetically lacking sphingomyelinase, which degrades sphingomyelin. In plasma, about $20 \%$ of lipoprotein phospholipids is sphingomyelin, most of which exists in VLDL and LDL ${ }^{1)}$. Sphingomyelin on the surface of LDL particles interferes in the reaction between LDL and LDL receptors ${ }^{2,3)}$ and promotes $\mathrm{LDL}$ aggregation ${ }^{2)}$. Aggregated LDL is captured by scavenger receptors of macrophages. These phenomena mean that sphingomyelin in LDL has pro-atherogenicity. As reported previously, the ingestion of plant stanol decreased the amount of sphingomyelin in LDL and lowered the aggregation susceptibility of LDL particles ${ }^{4)}$.

Sphingomyelin consists of ceramide (N-acyl sphingosine) and phosphoryl choline. Ceramide consists of sphingosine and a fatty acid (Fig.1). Ceramide and sphingosine also belong to sphingolipid. Ceramide is related to atherosclerosis. Meeusen et al. demonstrated that the number of plasma ceramides was associated with major adverse cardiovascular events in their cohort of 495 participants ${ }^{5}$. Concerning the molecular species of ceramides, they showed that N-palmitoyl sphingosine [Cer(16:0)], N-stearoyl-sphingosine [Cer(18:0)], and $\mathrm{N}$-nervonoyl-sphingosine [Cer(24:1)] are significantly predictive of a major cardiovascular outcome at 4 years of follow-up. Recently, ceramide has also been reported to be involved in the pathogenesis of type 2 diabetes mellitus. Neeland et al. reported that plasma ceramides, especially bound to saturated fatty acids, are related to visceral adiposity, insulin resistance, and the development of type 2 diabetes ${ }^{6}$. Fretts et al. also reported that plasma ceramide, especially $\operatorname{Cer}(16: 0)$, $\operatorname{Cer}(18: 0), \mathrm{N}$-arachidoyl-sphingosine [Cer(20:0)], and $\mathrm{N}$-behenoyl-sphingosine [Cer(22:0)], are associated with a higher risk of diabetes ${ }^{7}$.

In this issue of Journal of Atherosclerosis and Thrombosis, Kurano et al. reported that they measured ceramides and sphingosine, including dihydrosphingosine, a molecule that is closely related to sphingosine (Fig. 1), in each serum lipoprotein and lipoprotein-deleted fraction of healthy controls and patients with diabetes and compared the distribution of these sphingolipids between the two populations ${ }^{8)}$. Some of their patients with type 2 diabetic mellitus had complications related to cardiovascular diseases and diabetic nephropathy. They separated lipoproteins from the serum of all participants using sequential ultracentrifugation and measured sphingolipids in each fraction using the LC-MS/MS system. Although several researchers have mentioned the relationship between serum ceramides and atherosclerosis or metabolic deterioration in humans, the distribution of sphingolipids among lipoproteins is rarely observed. Therefore, the research of Kurano et al. is well noted. The important points they found are as follows: 1) the levels of ceramide, sphingosine, and dihydosphingosine in HDL were lower, whereas those in lipoproteindepleted fractions were higher in patients with diabetes; 2) the contents of sphingosine, $\operatorname{Cer}(16: 0)$ and N-lignoceroyl-sphingosine [Cer(24:0)] in the LDL fraction were higher in the group with diabetes, and levels of ceramides in LDL were negatively associated with the presence of cardiovascular diseases

Address for correspondence: Hiroshi Hayashi, Department of Internal Medicine, Tokyo Ariake University of Medical and Health Sciences, 2-9-1 Ariake, Koto-ku, Tokyo 135-0063, Japan E-mail: hhayashi@tau.ac.jp

Received: January 7, 2022 Accepted for publication: January 13, 2022

Copyright@2022 Japan Atherosclerosis Society

This article is distributed under the terms of the latest version of CC BY-NC-SA defined by the Creative Commons Attribution License. 


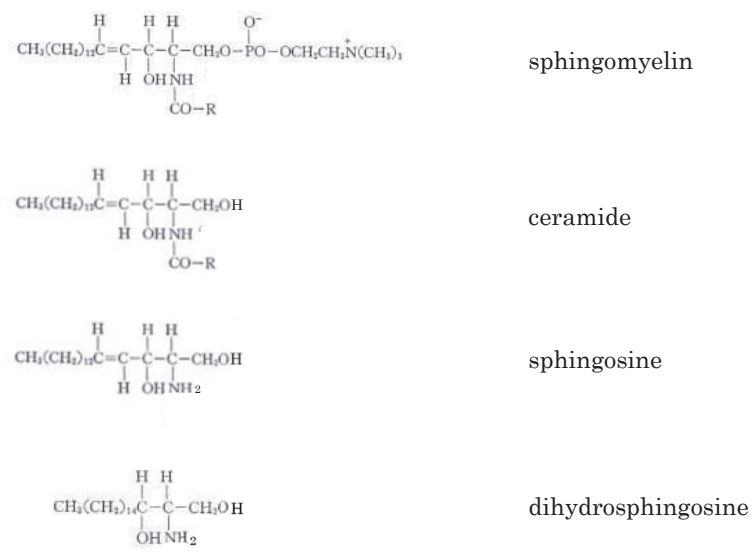

Fig. 1. The structures of sphingolipids are shown.

$\mathrm{CO}-\mathrm{R}$ denotes an acyl residue.

and stage 4 diabetic nephropathy in the group with diabetes; 3) each ceramide did not necessarily behave in the same mode, that is, the levels of $\mathrm{N}$-oleoylsphingosine [Cer(18:1)] in HDL were not significantly decreased in the group with diabetes.

The amount of ceramides measured in serum is related to atherosclerosis and type 2 diabetes, as mentioned above. Kurano et al. revealed a significant difference in the distribution of ceramides among lipoproteins and lipoprotein-deleted fractions of serum when comparing patients with diabetes to healthy subjects ${ }^{8)}$. Moreover, in patients with diabetes, ceramides increase in LDL, but they decrease in HDL compared to normal subjects. Surprisingly, the level of ceramides in the LDL of patients with diabetes is negatively related to cardiovascular events and diabetic nephropathy. Ceramides contained in lipoproteins behave differently in atherogenesis and metabolic conditions than those existing in lipoprotein-deleted fractions or bound to albumin. Kurano et al. described an interesting finding in their previous research that sphingosine 1-phosphate ( $\mathrm{S} 1 \mathrm{P})$, a sphingolipid, is anti-atherogenic when it is bound to HDL via apoprotein $\mathrm{M}$, but it exerts harmful effects on atherosclerosis when it is bound to albumin ${ }^{8}$. So, do the ceramides have the same effect as S1P? They calculated the ratio of ceramides to phospholipids, apolipoprotein B, or apolipoprotein A-I in each lipoprotein to elucidate the role of ceramides in lipoproteins but did not reach a definite conclusion in this research. This is an inevitable and important question and deserves further investigation. Kurano et al. found that $\operatorname{Cer}(18: 1)$ behaves a differently from other ceramides in the HDL of patients with diabetes $^{8)}$. It is reasonable to speculate that each ceramide affects metabolism differently depending on its molecular species not only in the whole serum but also in lipoproteins. More observations are expected.

Although the research of Kurano et al. is noteworthy, there are some concerns in their study. Some patients in the group with diabetes were prescribed statins. Statins naturally affect lipoprotein metabolism and may affect sphingolipid metabolism. They analyzed the data using various statistical methods and tried to cancel the confounding effects of statins, but it complicated the interpretation of the results. The number of subjects they measured was relatively small considering the mixed characters of the group. If they included more subjects for analysis, the results could become much clearer.

In conclusion, Kurano et al. opened the door to a new research field of sphingolipids, but the question of physiological and pathological roles of sphingolipids remains.

\section{Conflict of Interest}

None.

\section{References}

1) Nilsson $\AA$ and Duan R-D. Absorption and lipoprotein transport of sphingomyelin. J Lipid Res, 2006; 47: 154171

2) $\mathrm{Xu} X-X$ and Tabas I. Sphingomyelinase enhances low density lipoprotein uptake and ability to induce cholesteryl ester accumulation in macrophages. J Biol Chem, 1991; 266: 24849-24858

3) Gupta AK and Rudney H. Sphingomyelinase treatment of low density lipoprotein and cultured cells results in enhanced processing of LDL which can be modulated by sphingomyelin. J Lipid Res, 1992; 33: 1741-1752

4) Ruuth M, Äikäs L, Tigistu-Sahle F, Käkelä R, Lindholm

\section{Advance Publication Journal of Atherosclerosis and Thrombosis}


H, Simonen P, Kovanen PT, Gylling H and Öörni K. Plant stanol esters reduce LDL (low-density lipoprotein) aggregation by altering LDL surface lipids. The BLOOD FLOW Randomized Intervention Study. Arterioscler Thromb Vasc Biol, 2020; 40: 2310-2321

5) Meeusen JW, Donato LJ, Bryant SC, Baudhuin LM, Berger PB and Jaffe AS. Plasma ceramides. A novel predictor of major adverse cardiovascular events after coronary angiography. Arterioscler Thromb Vasc Biol, 2018; 38: 1933-1939

6) Neeland IJ, Singh S, McGuire DK, Vega GL, Roddy T, Reilly DF, Castro-Perez J, Kozlitina J and Scherer PE. Relation of plasma ceramides to visceral adiposity, insulin resistance and the development of type 2 diabetes mellitus: the Dallas Heart Study. Diabetologia, 2018; 61: 2570-2579

7) Fretts AM, Jensen PN, Hoofnagle AN, McKnight B, Howard BV, Umans J, Sitlani CM, Siscovick DS, King IB, Djousse L, Sotoodehnia N and Lemaitre RN. Plasma ceramides containing saturated fatty acids are associated with risk of type 2 diabetes. J Lipid Res, 2021; 62: 100119

8) Kurano M, Tsukamoto K, Sakai E and Yatomi Y. Differences in the distribution of ceramides and sphingosine among lipoprotein and lipoprotein-depleted fractions in patients with type 2 diabetes mellitus. J Atheroscler Thromb, 2021; 28: in press. doi: http://doi. org/10.5551/jat.63249 